\title{
Controlling Size and Fluorescence of Dye-Loaded Polymer Nanoparticles through Polymer Design
}

\author{
Vitalii Rosiuk, Anne Runser, Andrey Klymchenko, Andreas Reisch* \\ Laboratoire de Bioimagerie et Pathologies, CNRS UMR 7021, Université de Strasbourg, Faculté de \\ Pharmacie, 67401 Illkirch Cedex, France.
}

\begin{abstract}
Nanoprecipitation is a straightforward yet powerful technique to synthesize polymer nanoparticles loaded with various biologically active compounds or contrast agents. Particle formation in this approach is kinetically controlled and various assembly parameters have been used to control the size distribution and properties of the formed nanoparticles. Here, the influence of the nature of the polymer on the formation of nanoparticles in nanoprecipitation is studied systematically by varying its hydrophobicity and charge over a broad range. For this, methacrylate copolymers with different of types and fractions of hydrophobic, hydrophilic, and charged side groups are synthesized. Nanoprecipitation of these polymers shows that particle size increases with increasing global hydrophobicity of the polymers. At the same time, both hydrophilic and charged groups reduce particle size. In this way, we achieve control over particle size from $\sim 10$ to $200 \mathrm{~nm}$. Furthermore, the effect of the polymer nature on the photophysical properties of nanoparticles loaded with a fluorescent dye, a rhodamine B derivative with a bulky hydrophobic couterion (fluorinated tetraphenylborate), is studied. It is found that the hydrophobic/hydrophilic balance of the polymer modulates to a large extent the spectral properties and fluorescence quantum yield of the dye encapsulated at high concentration, which reflects changes in the dye aggregation within the polymer matrix. Thus, we show how polymer chemistry can tune kinetically controlled formation of nanoparticles and encapsulation of the load. The concepts introduced here should be valuable tools for the design of nanoparticles for imaging and drug-delivery applications.
\end{abstract}

Keywords

Polymer Nanoparticles, Nanoprecipitation, Nanoparticle Formation, Dye-loaded Polymer Nanoparticles, Fluorescent Nanoparticles 


\section{Introduction}

Polymer nanoparticles (NPs) have attracted increasing attention and gained considerable importance over the last two decades. ${ }^{1-3}$ In particular, the possibility to load them with various active or functional compounds has made them valuable tools in the biomedical field, for example in drug delivery ${ }^{4,5}$, or, when loaded with different contrast agents, ${ }^{6,7}$ in bioimaging. ${ }^{2,8}$ Several approaches have been developed for the synthesis of such loaded NPs, either using different types of emulsion polymerizations, or using different techniques for the assembly of preformed polymers. ${ }^{9}$ Among the latter, nanoprecipitation is a particular straightforward approach. ${ }^{10-12}$ The possibility to encapsulate very high amounts of load in the NPs in this way has been exploited, for example, for the synthesis of fluorescent dye-loaded polymer NPs and aggregation-induced emission NPs. ${ }^{13-15}$ In particular, the combination of charged fluorophores with hydrophobic bulky counterions could be used to prevent aggregation caused quenching and thus achieve particles with very high brightness, which can reach hundred times that of quantum dots. ${ }^{16,17}$ The high brightness and biocompatibility of these particles has enabled color coding of cells and the design of probes for intracellular imaging. ${ }^{18,19}$ At the same time, energy transfer between the encapsulated dyes is very efficient in these particles, which could be used to construct nanoantennas that allowed observing single molecules at very low excitation light intensities. $^{20}$

In nanoprecipitation, solutions of the polymers and/or the load in a water miscible organic solvent are added to an aqueous phase (Scheme 1). Interdiffusion of the two phases leads to a mixture in which the polymer/load are not soluble anymore, and thus to supersaturation of the polymer/load, which is the driving force of particle formation. As nanoprecipitation is a kinetically controlled process a precise control of the NP properties is challenging. ${ }^{1,21,22}$ Several assembly parameters as polymer concentration, ${ }^{21,23,24}$ type of solvent, ${ }^{23}$ type and speed of mixing, ${ }^{22,25,26}$ concentration and nature of stabilizing compounds ${ }^{27-29}$ have been found to exert a strong effect on particle formation and in particular on mean particle size and size distribution. ${ }^{10,30-32}$ Even so nanoprecipitation has been applied routinely to various polymers, including poly(lactic acid) (PLA), poly(lactic co glycolic acid) (PLGA), poly(alkyl cyanoacrylates) (PACA), poly(caprolactone) (PCL), and poly(methyl methacrylate) (PMMA), and their copolymers with poly(ethylene glycol) (PEG), the influence of polymer chemistry on particle formation in nanoprecipitation has been insufficiently investigated. ${ }^{10}$ In several cases the influence of the molecular weight of the polymer was evaluated, but did not show a major effect on particle size. ${ }^{33-35}$ We have recently determined the influence of the nature and the 
concentration of charged groups on the polymers on the size of NPs made through nanoprecipitation. ${ }^{19,36}$ Already small amounts of charged groups made it possible to assemble dye-loaded polymer NPs with sizes down to $10 \mathrm{~nm}$. By comparing dye-loaded polymer NPs made from PLGA, PMMA, and PCL with respect to their fluorescence properties, we could show how polymer chemistry can be used to improve their brightness but also to control the collective behavior of the fluorophores and thus particle blinking. ${ }^{16}$ However, more general studies on the role of polymer chemistry are largely missing to date.

The aim of the present study was to investigate systematically the influence of the nature of the polymer on NPs made through nanoprecipitation. To elucidate this question, we synthesized series of copolymers with different types and ratios of hydrophilic, hydrophobic, and charged monomers (Scheme 1). These copolymers were then used to assemble (dye-loaded) polymer NPs through nanoprecipitation. We were particularly interested in two aspects of the formed particles: their size and the photophysical properties of the encapsulated dyes. The latter reflect the organization and the environment of the dyes and thus characterize the encapsulation of the load. Particle size on the other hand depends on particle formation as a whole. Together, this work is the first attempt to provide a comprehensive picture of how polymer chemistry influences nanoprecipitation and how it can be used to control particle properties.
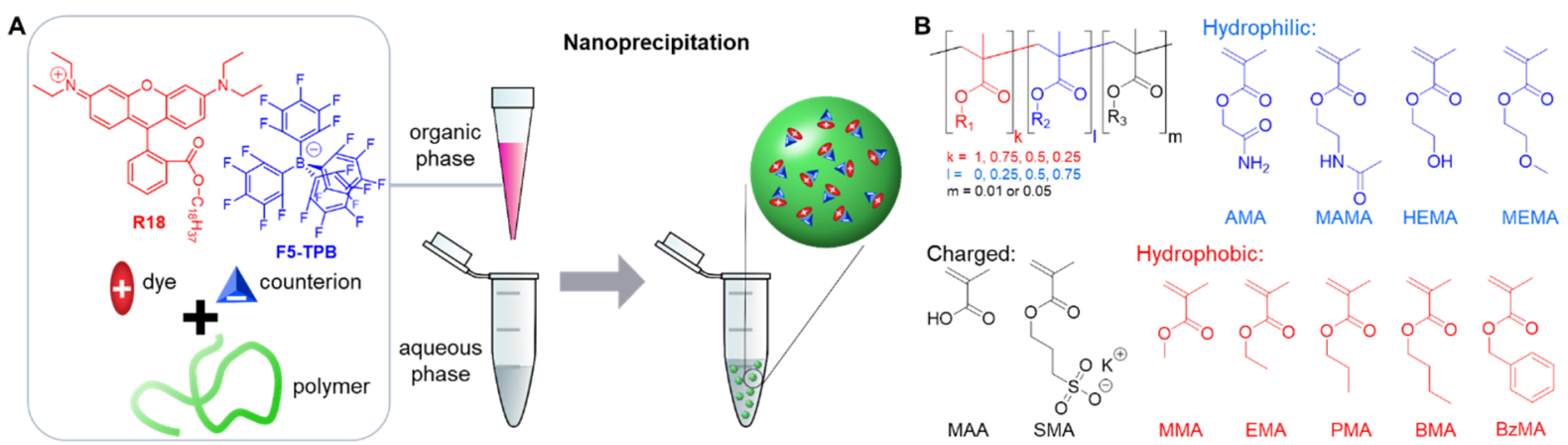

Scheme 1. (A) Schematic view of the assembly of dye-loaded polymer NPs through nanoprecipitation. The structure of the dye-counterion pair used in this study is given. (B) Overview of the monomers used in the design of copolymers. Typically, one charged, one hydrophilic, and one hydrophobic monomer were combined in different ratios to synthesize the copolymer. 


\section{Materials and Methods}

\section{Materials}

Methyl methacrylate (MMA, 99\%), methacrylic acid (MAA, 99\%), 2-aminoethyl methacrylate hydrochloride (90\%), 3-sulfoproppyl methacrylate potassium salt (SMA, 98\%), ethyl methacrylate (EMA, 99\%), n-propyl methacrylate (PMA, 97\%), butyl methacrylate (BMA, 99\%), benzyl methacrylate (BzMA, 96\%), hydroxyethyl methacrylate (HEMA, $\geq 99 \%$ ), ethylene glycol methyl ether methacrylate (MEMA, 99\%), iodoacetamide (99\%), acetanhydiride (> 99\%) were purchased from Sigma-Aldrich. Acetonitrile (HPLC grade), diisopropylethylamine (DIPEA, >98\%), dimethylformamide (DMF, analytical grade), potassium carbonate $\left(\mathrm{K}_{2} \mathrm{CO}_{3}, \geq 99.5 \%\right)$, and dimethylsulfoxide (DMSO, analytical grade) were obtained from Fisher-Scientific, dichloromethane (DCM, $\geq 99.8 \%$ ) from CarloErba and methanol (HPLC grade) from VWR. Monomers were purified using column chromatography or re-crystallization. Azobis isobutyronitrile (Aldrich, $\geq 98 \%$ ) was recrystallized twice from ethanol. The other compounds were used as received. Milli-Q water (Millipore) was used in all experiments.

R18/F5-TPB was synthesized from rhodamine B octadecyl ester perchlorate (Aldrich, >98.0\%) and lithium tetrakis(pentafluorophenyl)borate ethyl etherate (AlfaAesar, 97\%) through ion exchange followed by purification through column chromatography as described previously. ${ }^{17,18}$

2-acetamidoethyl methacrylate (MAMA): $1.59 \mathrm{~g}$ of 2-aminoethyl methacrylate hydrochloride (8.6 mmol) were added to $20 \mathrm{~mL}$ of $\mathrm{DCM}$ in a $100 \mathrm{~mL}$ round bottom flask, then $2.43 \mathrm{~mL}$ of DIPEA (15 mmol) were added. $1.38 \mathrm{~g}$ of acetanhydride $(14 \mathrm{mmol})$ were added dropwise to the mixture and the reaction was left for 30 minutes. The reaction mixture was extracted with $\mathrm{HCl}$ $(1 \mathrm{M})$ then with aqueous solutions of $\mathrm{K}_{2} \mathrm{CO}_{3}$ and finally with distilled water. After evaporating the organic phase $1.37 \mathrm{~g}$ (8 mmol) of a clear liquid were obtained (yield 90\%). ${ }^{1} \mathrm{H}$ NMR (400 MHz, DMSO-d $d) \delta(\mathrm{ppm}):$ 8,03 (s, 1H), 6,06 (s, 1H), 5,69 (s, 1H), 4,08 (t, 2H), 3,32 (t, 2H), $1,88(\mathrm{~s}, 3 \mathrm{H}), 1,82(\mathrm{~s}, 3 \mathrm{H}) .{ }^{13} \mathrm{C}$ NMR $(500 \mathrm{MHz}, \mathrm{MeOD}) \delta$ (ppm) 173.5, 168.7, 137.6, 126.4, 64.3, 39.5, 22.5, 18.3. HRMS (m/z): [M] ${ }^{+}$calcd. for $\mathrm{C}_{8} \mathrm{H}_{14} \mathrm{NO}_{3}, 172.0968$; found, 172.0965 .

2-amino-2-oxoethyl methacrylate (AMA): $3.17 \mathrm{~g}$ of methacrylic acid (37 mmol), $7.72 \mathrm{~g}$ of iodoacetamide (42 mmol), and $6,9 \mathrm{~g}$ of $\mathrm{K}_{2} \mathrm{CO}_{3}(50 \mathrm{mmol})$ were added successively to $30 \mathrm{~mL}$ of DMF in a $100 \mathrm{~mL}$ round bottom flask. The mixture was heated at $50{ }^{\circ} \mathrm{C}$ over 18 hours. After this time the mixture was filtered and extracted with brine/ethyl acetate and two times distilled water/ethyl acetate. Drying under reduced pressure afforded $1.98 \mathrm{~g}$ of a white solid (yield 38\%). 
${ }^{1} \mathrm{H}$ NMR (400 MHz, DMSO- $\left.d_{6}\right) \delta(\mathrm{ppm})$ 7,43 (s, 1H), 7,24 (s, 1H), 6,16 (s, 1H), 5,74 (s, 1H), 4,52 (s, 2H), 1,92 (s, 3H). ${ }^{13} \mathrm{C}$ NMR (500 MHz, MeOD) $\delta$ (ppm) 172.6, 167.9, 137.1, 127.2, 63.4, 18.3. HRMS (m/z): [M] $]^{+}$calcd. for $\mathrm{C}_{6} \mathrm{H}_{10} \mathrm{NO}_{3}, 144.0655$; found, 144.0652.

\section{Polymer synthesis}

Polymers were synthesized through free radical polymerization. Monomers were dissolved in DMSO and mixed at the desired ratio in a $1.5 \mathrm{~mL}$ Eppendorf tube. 0.01 eq of AIBN were added and the mixture was placed in a shaker preheated to $70{ }^{\circ} \mathrm{C}$. Once the conversion reached around $25 \%$, the reaction was stopped and the polymers reprecipitated twice in methanol/water mixtures. Detailed information about synthesized polymers is given in the Supporting Information.

We used NMR-spectroscopy for evaluating the polymerization and the resulting polymers: NMR spectra were recorded at $20{ }^{\circ} \mathrm{C}$ on a BrukerAvance III 400 spectrometer. First, the feed composition is analyzed. Second, the conversion of the polymerization is monitored. Finally, the dried polymers are analyzed to obtain the composition of the synthesized polymers.

As an example the copolymerization of $49.5 \mathrm{~mol} \%$ ethyl methacrylate, $49.5 \mathrm{~mol} \% 2$ hydroxyethyl methacrylate, and $1 \mathrm{~mol} \%$ methacrylic acid is given:

Poly(ethyl methacrylate-co-2-hydroxyethyl methacrylate-co-methacrylic acid): Monomers were dissolved in DMSO at a concentration of $1 \mathrm{M}$. The three solutions were then mixed in a $1.5 \mathrm{~mL}$ eppendorf tube at the desired ratio to give a total volume of $1 \mathrm{~mL}: 0.495 \mathrm{~mL}$ of ethyl methacrylate solution, $0.495 \mathrm{~mL}$ of 2-hydroxyethyl methacrylate solution, and $0.01 \mathrm{~mL}$ of methacrylic acid solution. NMR analysis gave a feed composition of $50.5 \mathrm{~mol} \%$ of HEMA, $48.5 \mathrm{~mol} \%$ of EMA and $1 \mathrm{~mol} \%$ of MAA. 0.01 eq of AIBN in DMSO (40 mg/mL) were then added and the Eppendorf tube was placed in a shaker preheated to $70{ }^{\circ} \mathrm{C}$. Samples were drawn and analyzed by NMR to monitor conversion. Once the conversion reached 25\% (after $28 \mathrm{~min}$ ) the reaction was stopped by quickly cooling the Eppendorf tube in the fridge. The reaction mixture was added dropwise to a methanol:water (1:4) mixture. After filtration the precipitate was redissolved in a small amount of acetone and reprecipitated twice in methanol-water mixtures. The obtained polymer was dried under vacuum to give $21 \mathrm{mg}$ of a white solid (global yield $17 \%$ ). NMR gave $50 \mathrm{~mol} \%$ of HEMA and $50 \mathrm{~mol} \%$ of EMA (MAA is not clearly identifiable in the final polymer). Molar mass by size exclusion chromatography (SEC, Shimadzu, equipped with triple detection based on light scattering, refractive index and viscosimetry, using 3 PLgel mixte B columns as stationary and tetrahydrofuran with $0.01 \mathrm{M}$ tetrabutylammonium tetrafluoroborate as mobile phase with a flow rate of $1 \mathrm{~mL} . \mathrm{min}^{-1}$ ) for 
Poly(methyl methacrylate- co-methacrylic acid): $1 \%$ MAA: $\mathrm{M}_{\mathrm{w}}=38100, \mathrm{M}_{\mathrm{w}} / \mathrm{M}_{\mathrm{n}}=1.35 ; 5 \%$ MAA: $\mathrm{M}_{\mathrm{w}}=46800, \mathrm{M}_{\mathrm{w}} / \mathrm{M}_{\mathrm{n}}=1.34$.

For simplicity the MAA percentage will be omitted from the global composition, i.e. a $1 \%$ MAA, 49.5\% EMA, 49.5\% HEMA copolymer will be noted 1\% MAA, 50\% EMA, 50\% HEMA. ChemDraw 15 Professional was used to calculate octanol-water partition coefficients ( $\log \mathrm{P}$ values) for 4 repeat units.

\section{Turbidimetry}

For turbidimetry measurements the optical density (absorbance) was measured at $400 \mathrm{~nm}$ using a Cary 4000 Scan ultraviolet-visible spectrophotometer (Varian). $800 \mu \mathrm{L}$ of polymer solution in acetonitrile/acetone ( $4: 1 \mathrm{v} / \mathrm{v})$ were placed in a quartz cuvette. Defined volumes of water were then added to this solution followed by mixing and recording of the absorbance. To determine the onset of precipitation, the absorbance was plotted $v s$ the water fraction of the solution.

Preparation of NPS

Solutions of the polymers at $2 \mathrm{~g} / \mathrm{L}$ in acetonitrile/acetone ( $4: 1 \mathrm{v} / \mathrm{v}$, up to $15 \mathrm{vol} . \%$ methanol for polymers with high content of hydrophilic monomers, in special cases with addition of some amount of DMSO extra acetone and methanol) were prepared. For assembly of dye loaded NPs these solutions contained $10 \mathrm{wt} \%$ (relative to the polymer) of the R18/F5-TPB dye salt. These solutions were then added quickly and under stirring (shaking) using a micropipette to a 10fold volume excess of water or $\mathrm{pH} 7.4$ phosphate buffer at $21^{\circ} \mathrm{C}$. The particle solution was then quickly diluted five-fold in water.

Characterization of NPs:

Dynamic light scattering $(D L S)$. Size and $\zeta$-potential measurements were performed on a Zetasizer Nano series ZSP (Malvern Instruments S.A.). For size determination each sample was measured 10 times with a run length of $10 \mathrm{~s}$ each. The volume average values were used, which are determined by the Zetasizer software (Malvern) based on Mie theory. For $\zeta$-potential determination three successive measurements combining electrophoretic mobility and Laser Doppler Velocimetry with $>10$ runs each were carried out with an applied potential of $\pm 150 \mathrm{~V}$. Absorption and emission spectra were recorded on a Cary 4000 Scan ultraviolet-visible spectrophotometer (Varian) and an Edinburgh FS5 spectrofluorometer equipped with a thermostated cell compartment, respectively. Excitation was performed at $530 \mathrm{~nm}$.

Quantum yields (QYs) were determined using a simplified relative method: ${ }^{37,38}$ Absorption and emission spectra $\left(\lambda_{\mathrm{ex}}=530 \mathrm{~nm}\right)$ of the dye-loaded NPs in water and of rhodamine 101 in ethanol 
as reference were recorded for solutions with peak absorbances below 0.1. The QYs were then calculated according to:

$Q Y_{N P}=Q Y_{r 101} \frac{F_{N P} A_{N P, 530 n m} n_{H_{2} O}^{2}}{F_{r 101} A_{r 101,530 n m} n_{E t O H}^{2}}$

where $F_{N P}$ and $F_{r 101}$ corresponds to the emission intensity integrated over the whole emission wavelength range of the NP and rhodamine 101 solutions, respectively, $A_{x, 530 n m}$ to their absorbance at the excitation wavelength, and $\mathrm{n}$ to the refractive indices of the solvents $(1.33$ for water and 1.36 for ethanol). QY $\mathrm{Y}_{\mathrm{r} 101}$ is the quantum yield of the standard, rhodamine 101, which was taken to be $0.95 .^{37,38}$

\section{Results and Discussion}

\section{Polymer design}

Copolymers were synthesized through combination of three types of methacrylate monomers: a hydrophilic monomer, bearing either $\mathrm{OH}$ or amide groups, a hydrophobic monomer with different length of the alkyl side chain or a benzyl group, and the (potentially) charged methacrylic acid and sulfopropyl methacrylate (MAA, SMA, Scheme 1). The methacrylic acid was used at low fractions of either 1 or $5 \mathrm{~mol} \%$, the sulfonate monomer only at $1 \mathrm{~mol} \%$, which has proven sufficient to control particle size. ${ }^{19,36}$ In the reaction mixture, the fraction of the hydrophilic monomers was varied from 0 to $75 \%$. Polymerizations were stopped once the conversion reached about $20 \%$ in order to limit heterogeneities of the composition of the copolymers. The resulting polymers were analyzed with respect to their composition using NMR spectroscopy, based on the protons of the $\mathrm{CH}_{2}$ groups $\alpha$ and $\beta$ to the ester group (Figure 1, Supporting Information Table S1). In general, the obtained ratio of the hydrophobic and hydrophilic monomers in the purified polymers corresponded to the feed ratio (within $\pm 2 \%$ ). In this way, a set of over 50 amphiphilic copolymers with varying types and ratios of the different groups were obtained (Table S1). 


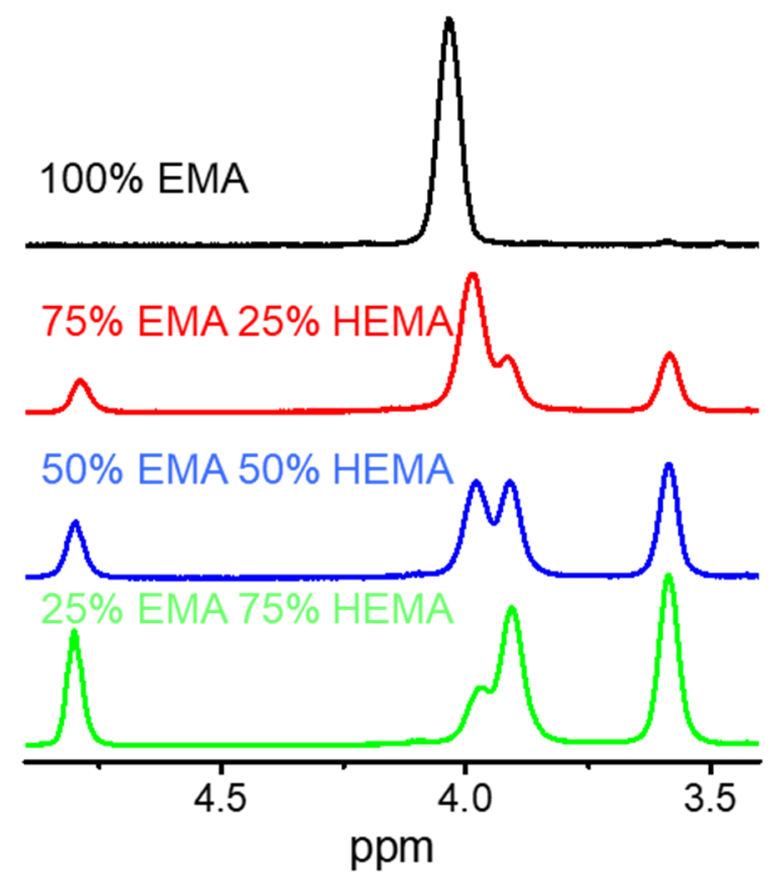

Figure 1. ${ }^{1} \mathrm{H}-\mathrm{NMR}$ spectra of polymers with different EMA/HEMA ratios. The region used for the determination of the ratios of comonomers is shown. Spectra were recorded in $\mathrm{CDCl}_{3}$ for $100 \% \mathrm{EMA}$ and in DMSO- $d_{6}$ for the others.

\section{$\underline{\text { Hydrophobicity of copolymers }}$}

The resulting set of amphiphilic polymers was first analyzed with respect to their hydrophobicities/ hydrophilicities. A key parameter in nanoprecipitation is the solubility of the polymer in mixtures of organic solvent and water, and especially the water content at which the polymers start aggregating, as it determines the obtained supersaturation, which is the driving force of particle formation. We measured this critical water content through turbidity measurements by adding increasing amounts of water to solutions of the different polymers in acetone/acetonitrile mixtures. Results showed a continuous decrease of the critical water fraction with increasing fraction of hydrophobic monomer (Figure 2A). For a given ratio of hydrophilic to hydrophobic monomer, this parameter decreased in the following order: MMA $>$ EMA > PMA > BMA > BzMA (Figure 2). The critical water fractions correlated well with calculated octanol-water partition coefficients (log $\mathrm{P}$ values) for the different copolymer compositions (Figure $2 \mathrm{C}$, Figure S1), indicating that the global hydrophobicity determines the solubility of the copolymers in organic solvent / water mixtures, and thus the starting point of the precipitation. 

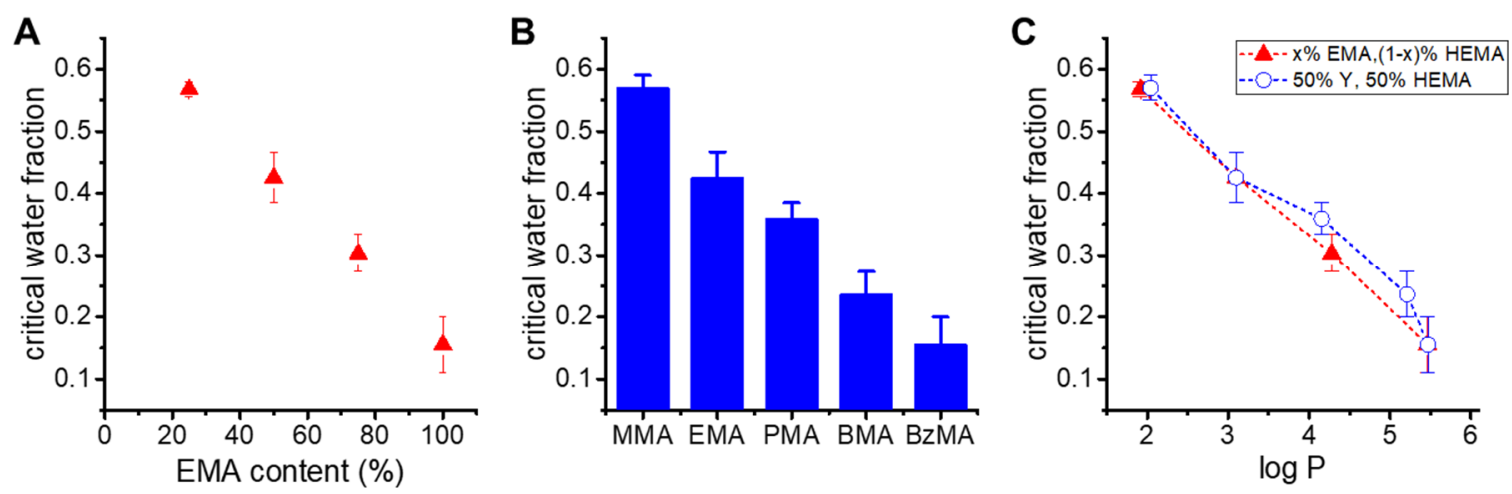

Figure 2. Critical water fraction (volume fraction) leading to precipitation as determined from turbidity measurements as a function of: (A) EMA fraction in a EMA/HEMA/MAA copolymer with 1 mol\% MAA; (B) the hydrophobic monomer in copolymers with 50 mol\% HEMA and 1 mol\% MAA. (C) Critical water fraction for the two series of copolymers vs calculated log P values. Error bars give uncertainty of onset of turbidity. Dashed lines are guides to the eye.

\section{Influence of copolymer composition on NP size and charge}

The copolymers were then used for the assembly of nanoparticles through nanoprecipitation. For this, they were dissolved in a water miscible organic solvent (acetonitrile/acetone 4:1, with a small amount of methanol for the least hydrophobic polymers). These solutions were then added quickly to a tenfold excess of an aqueous solution as the nonsolvent, followed by further dilution, where needed (Scheme 1). The sizes of the resulting NPs were analyzed by dynamic light scattering (DLS), which showed monomodal distributions with PDI values $<0.2$ for practically all formulations, and confirmed by transmission electron microscopy (Figure S5). For all combinations of monomers, increasing the fraction of the hydrophilic monomer led to a continuous decrease in the size of the resulting particles (Figure $3 \mathrm{~A}$ ). The polymers made only from the hydrophobic monomers (and 1\% of MAA) yielded particles of size $>100 \mathrm{~nm}$. For the highest fractions of hydrophilic monomers $(75 \mathrm{~mol} \%)$, the sizes decreased to less than $40 \mathrm{~nm}$. Going from 1 to $5 \mathrm{~mol} \%$ of the charged MAA yielded a decrease in size of about $20 \mathrm{~nm}$, yet a bit less for the least hydrophobic systems. For the highest ratios of hydrophilic monomers this thus lead to particle sizes of 20 to $30 \mathrm{~nm}$. A further decrease in size could be obtained by using the sulfonate bearing SMA, even at 1 mol\%, leading to particle sizes close to $10 \mathrm{~nm}$ (Figure $4 \mathrm{~A}$ ). 

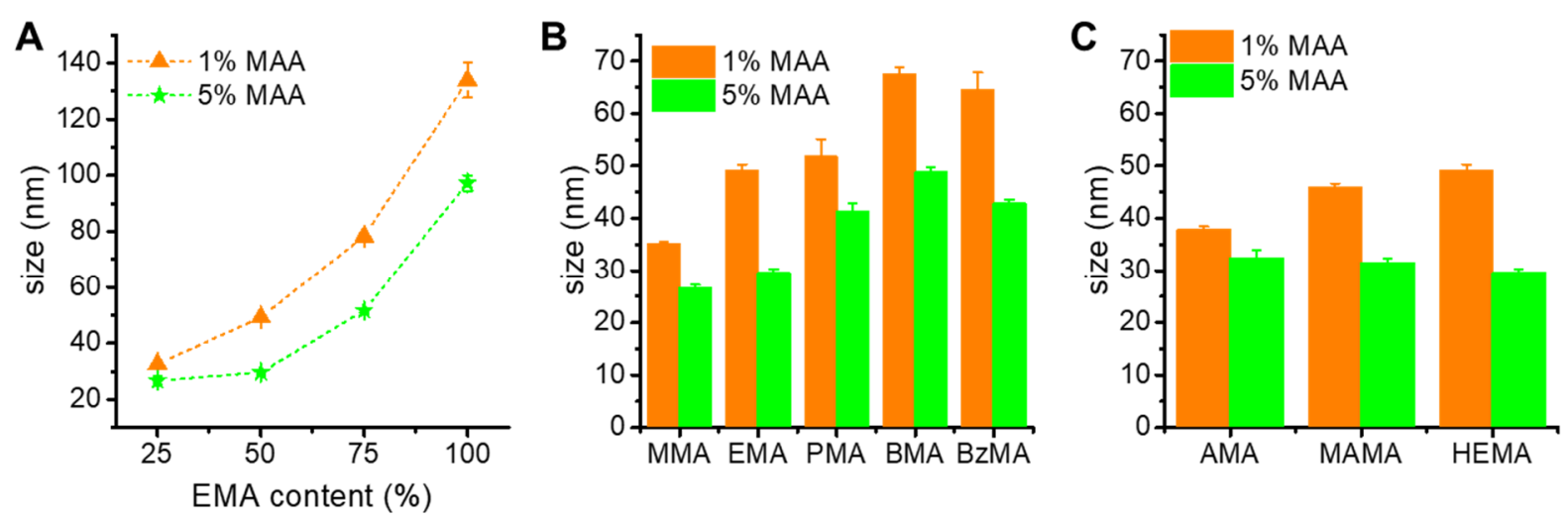

Figure 3. Influence of copolymer composition on size of particles obtained through nanoprecipitation: (A) Influence of ratio of monomers for HEMA/EMA/MAA copolymers. (B) Influence of the type of hydrophobic monomer for copolymers with HEMA (50 mol\%) and MAA. (C) Influence of the type of hydrophilic monomer for copolymers with EMA (50 mol\%) and MAA. Sizes were determined through dynamic light scattering, and give the average of 3 independent measurements of the volume weighted diameter. Error bars give standard error of the mean.

The type of hydrophobic monomer also influenced the size of the NPs (Figure 3B, for HEMA as hydrophilic monomer and a 1:1 ratio). For practically all ratios the size increased in the order MMA $<$ EMA $<$ PMA $<$ BMA $\sim$ BzMA, thus following the increase in hydrophobicity of the monomer. Similarly, the type of hydrophilic monomer also had an influence on the size: For a given hydrophobic monomer and monomer ratio, the size increased in the order AMA $<$ MAMA $<$ HEMA( $<$ MEMA) (Figure 3C), again in line with increasing hydrophobicity. However, differences between AMA, MAMA and HEMA copolymers were relatively small and the trend could even be inversed (or at least vanished) for $5 \mathrm{~mol} \%$ of charged monomer.

Variation of the polymer composition also influenced the $\zeta$-potential of the formed particles, which was negative for all systems studied here (Tables S3 and S4). Increasing the fraction of hydrophobic monomer resulted in a more negative $\zeta$-potential for the most hydrophobic compositions. Moreover, for a given hydrophilic to hydrophobic ratio the $\zeta$-potential became more negative with increasing hydrophobicity of the alkyl methacrylate monomer, going from $-17 \mathrm{mV}$ for MMA to $-39 \mathrm{mV}$ for BMA and BzMA (Table S4). As expected, going from 1 to $5 \%$ of carboxylate groups also led to a more negative $\zeta$-potential, going for example for 1:1 HEMA/EMA copolymers from $-18 \mathrm{mV}$ for $1 \mathrm{~mol} \%$ MAA to $-27 \mathrm{mV}$ for $5 \mathrm{~mol} \%$ MAA. Even though some of the NPs had $\zeta$-potentials less negative than $-30 \mathrm{mV}$, 
no signs of aggregation were seen in DLS over the time-frame of the experiments (several hours).
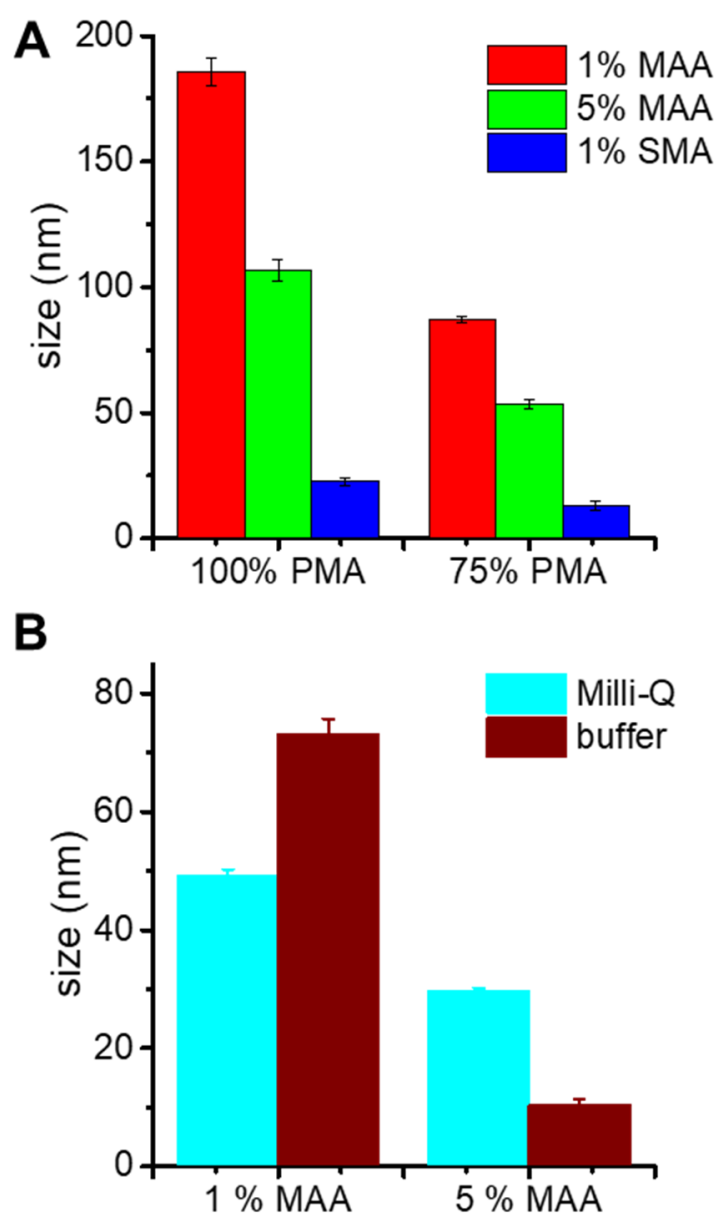

Figure 4. Influence of the nature of the charged group and of the precipitation medium: (A) Size of NPs made from copolymers containing a small fraction of charged monomer (MAA or SMA) and PMA or 75\% PMA with 25\% HEMA. Prepared in Milli-Q water. (B) Size of NPs formed in either Milli-Q water or $20 \mathrm{mM}$ phosphate buffer at $\mathrm{pH}$ 7.4, from HEMA/EMA/MAA copolymers with 50\% EMA content and 1 or $5 \mathrm{~mol} \%$ of charged monomer.

The precipitation medium influenced the size of the obtained NPs depending on the amount of charged monomer: In the case of 5 mol\% MAA the use of phosphate buffer (pH 7.4) led to a decrease in size compared to Milli-Q water as precipitation medium. In the case of 1 mol\% MAA, however, the opposite was true (Figure $4 \mathrm{~B}$ ). At low (1 mol\%) fraction of MAA it is likely that the degree of ionization does not significantly differ between both media (at the end of the first precipitation step: $\left.[\mathrm{COOH}]=0.0002 \mathrm{~mol} \cdot \mathrm{L}^{-1}, \log [\mathrm{COOH}]=-4.7\right)$. The higher 
ionic strength in the buffer thus decreases the repulsion between polymers/particles, leading to larger particles. At higher $(5 \mathrm{~mol} \%)$ MAA fraction $\left([\mathrm{COOH}]=0.001 \mathrm{~mol} \cdot \mathrm{L}^{-1}, \log [\mathrm{COOH}]=-\right.$ 4, <pKa), the ionization should be increased in buffer at higher $\mathrm{pH}$, increasing the number of charged groups per polymer and in consequence the repulsion between chains and/or particles.

\section{$\underline{\text { Influence of copolymer composition on photophysical properties }}$}
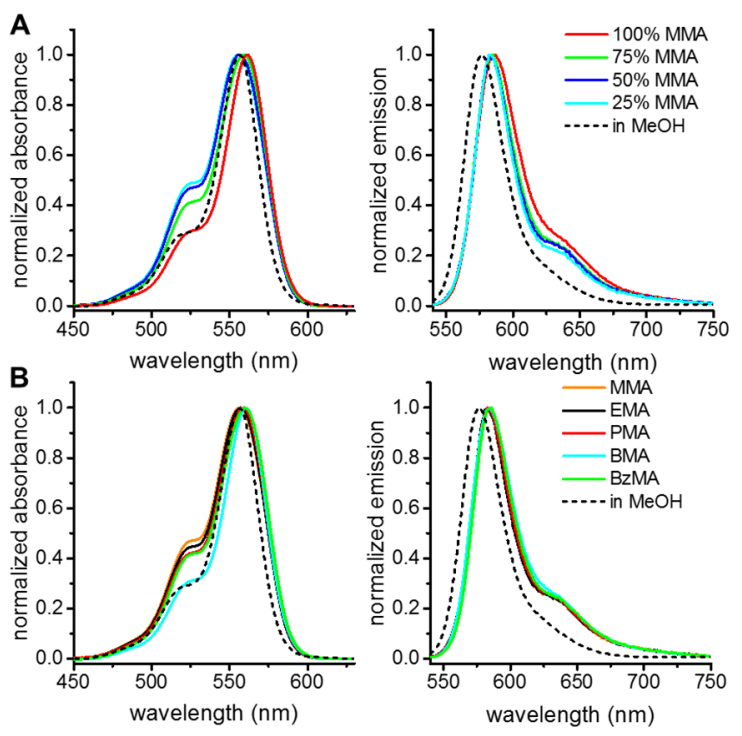

Figure 5. Normalized absorption (left) and emission (right) spectra of NPs made (A) from HEMA/MMA copolymers with different ratios (1 mol\% MAA) and (B) from copolymers with 50\% HEMA and different hydrophobic comonomers (1 mol\% MAA). NPs were loaded with 10 wt\% of R18/F5-TPB. Excitation was performed at $530 \mathrm{~nm}$.

We then analyzed the influence of the copolymer composition on the photophysical properties of dyes encapsulated in these NPs. For this, $10 \mathrm{wt} \%$ (relative to the polymer) of the salt of a hydrophobic rhodamine B derivative (R18) with a bulky hydrophobic counterion (perfluorinated tetraphenyl borate, F5-TPB) were added to the organic solution of the polymers. The use of such counterions has been shown to strongly reduce aggregation caused quenching of dyes upon their encapsulation in polymer NPs and thus allowed to assemble very bright fluorescent NPs. ${ }^{14,16,17}$ Furthermore, it resulted in $\geq 95 \%$ encapsulation efficiency of the dyes. ${ }^{36,39}$ The polymer/dye salt solutions were then used for the formation of NPs through nanoprecipitation in Milli-Q water as before. Absorption spectra showed an increasing relative intensity of the shoulder around $530 \mathrm{~nm}$ with respect to the absorption maximum for increasing fraction of hydrophilic monomer, accompanied by a slight blue shift of the absorption maxima 
(Figure 5A). In NPs made from polymers containing only the hydrophobic monomer (and MAA) the ratio of the shoulder to the maximum was practically the same as for the fluorophore in organic solvent (methanol). This indicates decreasing aggregation of the fluorophores inside the NPs with increasing hydrophobicity of the polymers. The type of hydrophobic monomer influenced how the intensity of the shoulder increased with the fraction of hydrophilic monomer (Figures 5B and S2).
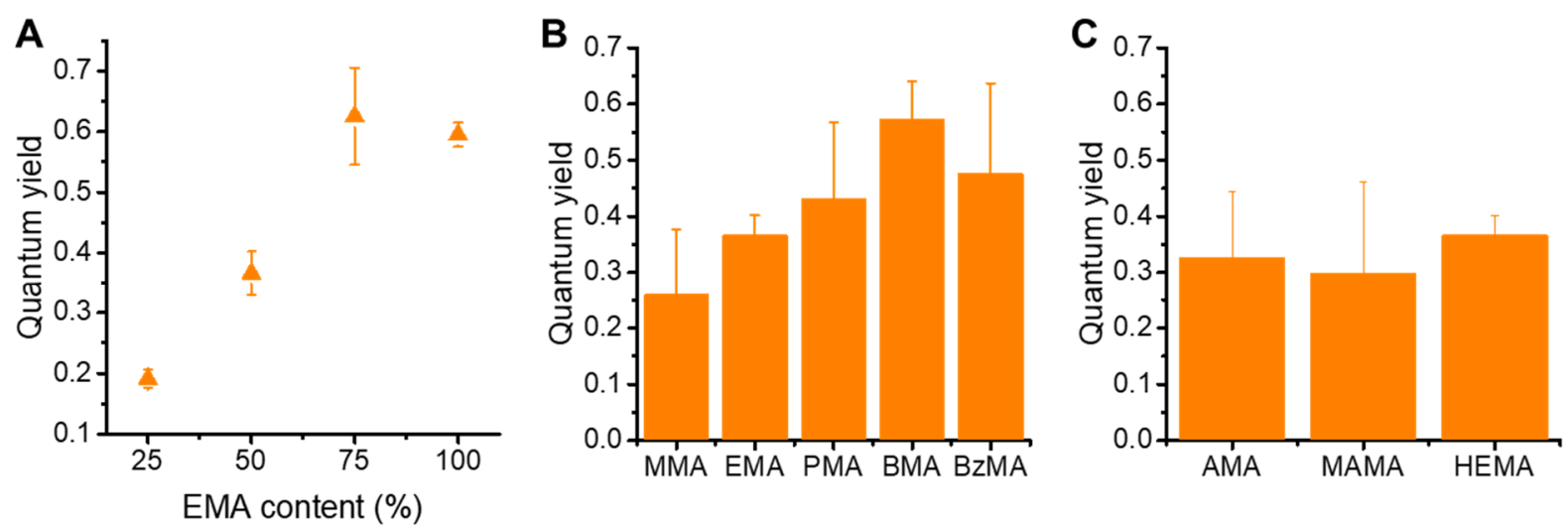

Figure 6. Fluorescence quantum yields of NPs made from different copolymers and loaded with 10 wt\% of R18/F5-TPB. (A) Quantum yields vs EMA content for copolymers with HEMA and 1 mol\% of MAA. (B) Influence of type of hydrophobic monomer for copolymers with HEMA (50 mol\%) and MAA (1 mol\%). (C) Influence of type of hydrophilic monomer for copolymers with EMA (50 mol\%) and MAA (1 mol\%). NPs were prepared in Milli-Q water. Error bars give standard error of the mean over at least three independent experiments.

Emission spectra of the encapsulated dye showed a slight red shift of the emission maximum with respect to its solution in methanol and the appearance of a small shoulder around $630 \mathrm{~nm}$. With decreasing fraction of hydrophobic monomer in the polymers the emission spectra became slightly narrower and blue shifted (Figure 5A). On the other hand, the fluorescence quantum yield (QY) increased strongly with increasing fraction of hydrophobic monomer in the polymers (Figure 6). For example, in the case of EMA/HEMA copolymers, the QY increased from less than $20 \%$ for 25 mol\% EMA content to over $60 \%$ for 75 mol\% EMA. A further increase in EMA content did not lead to a further increase in the QY. The nature of the hydrophobic monomer also influenced significantly the QY values. For a 1:1 ratio of HEMA and the hydrophobic monomer, the QY increased in the order MMA $<$ EMA $<$ PMA $<$ BzMA $\sim$ BMA. However, the actual dependence of the QY on the 
monomer ratio was quite different depending on the type of hydrophobic monomer (Figure S3): While for MMA and BzMA a continuous increase with hydrophobic monomer content was observed, the QYs went through a maximum for EMA and PMA at 75 mol\% of hydrophobic monomer. For BMA no clear trend was observed. The type of hydrophilic monomer had no significant influence on the QYs (Figure 6C). In the case of QY measurements, we observed a particular variation of the experimental errors (Fig. S3). Indeed, the reproducibility was quite good for the systems with the lowest and highest content of hydrophobic monomers, leading to a standard error of the mean of typically less than $10 \%$ (relative). The QY variability within repeated experiments were much larger for intermediate monomer concentrations and sometimes even two groups of QY values were observed with the standard error of the mean reaching $>40 \%$ (relative).

\section{$\underline{\text { Discussion }}$}

Thus, the nature of the polymer used for nanoprecipitation has a strong influence on the obtained NPs. In Figure 7, the size and the quantum yield of the assembled dye-loaded polymer particles are plotted versus the global hydrophobicity (calculated $\log \mathrm{P}$ ) of the polymer used for their preparation. Concerning the particle size there seemed to be three major influences of the polymer composition: (i) The size increased, in general, with increasing global hydrophobicity of the polymer. (ii) For equal global hydrophobicities, the sizes were smaller for polymers with a larger percentage of charged monomer (MAA). At the same time, the size of NPs made from

polymers bearing $1 \mathrm{~mol} \%$ MAA increased faster with increasing hydrophobicity than for polymers bearing 5 mol\% MAA. (iii) Polymers containing only the hydrophobic monomer and charged MAA, but no hydrophilic monomer, gave significantly larger particles. The size of these particles depended less clearly on polymer hydrophobicity. 



Figure 7. (A) Size and (B) quantum yield of nanoparticles plotted versus calculated log $P$ values of the polymers used for their preparation. In (A) results are given for polymers with 1 (triangles) and 5 (stars) mol\% of MAA. Half-filled symbols are used for the NPs made from polymers containing hydrophilic and hydrophobic monomers ( $\leq 75 \% \mathrm{hp}$ ) and filled symbols are used for those containing only the hydrophobic monomer (99 or $95 \% \mathrm{hp}$ ) and MAA. In (B) all polymers contained 1 mol\% MAA, and 10 wt\% R18/F5-TPB were encapsulated. Each series with a given hydrophobic monomer is shown in a distinct color. All NPs were prepared in Milli-Q water. Error bars give standard error of the means over at least three measurements.

In order to understand how the nature of the polymer influences the properties of particles, the mechanism of particle formation in nanoprecipitation has to be considered (Figure 8$)^{1,11,21}$ : Upon addition of the organic phase to the aqueous phase interdiffusion of the solvents leads to 
an increasing water content of the organic phase. At some point the polymer (and/or the dye salt) are not soluble anymore in the mixture, leading to supersaturation. This will in turn be the driving force for nucleation. The formed nuclei will then grow through incorporation of polymers and dye salts. In the absence of suitable stabilization, aggregation of the particles will occur. Particle formation in nanoprecipitation is under kinetic control, and so the rates of the different steps will define particle properties as the size distribution. Theoretical approaches are often based on theories developed for crystallization, e.g. classical nucleation theory. ${ }^{1}$ Smallest particles will be formed for high nucleation rates, low growth rates, and very low aggregation rates.

The nucleation rate can be estimated by ${ }^{1,21,22}$ :

$$
J=K_{1} \exp \left(-\frac{16 \pi \gamma^{3} v^{2}}{3 k^{3} T^{3}[\ln (S)]^{2}}\right)
$$

where $\gamma$ is the surface tension of the formed nuclei and $v$ the molar volume. The supersaturation $\mathrm{S}$ is given by:

$$
S=\frac{C}{C_{\text {sol }}}
$$

where $\mathrm{c}$ is the actual polymer concentration (in fact, the local effective concentration) and $\mathrm{c}_{\mathrm{sol}}$ the polymer solubility. ${ }^{1,22}$ Thus, at constant temperature (and molar volume of the polymer), the nucleation rate depends largely on supersaturation and surface tension. In particular, it increases with increasing supersaturation and decreases with increasing surface tension. The growth rate also increases with supersaturation. However, different growth types and dependencies have been postulated and observed depending on precipitation conditions and supersaturation regime. ${ }^{1,21,22,40}$

Here, the same conditions of temperature, concentration, and preparation (mixing) have been used for all polymers. The observed differences can thus be attributed to the differences in polymer chemistry through a combination of several effects, assuming that the charged and hydrophilic groups are preferentially situated at the surface of the particles: (i) Increase in the global hydrophobicity and lower fraction of hydrophilic groups should lead to an increase in surface tension of formed nuclei and thus a decrease in nucleation rate. In particular, NPs made from polymers containing only the hydrophobic monomer (and MAA) are much bigger than those containing at least a small fraction of hydrophilic monomer, which is able to reduce surface tension of forming nuclei. This also suggests a high importance of the nucleation rate. (ii) The general trend of increasing size with increasing global hydrophobicity indicates that the ratio of particle growth rate to nucleation rate increases with supersaturation. This is in 
agreement with earlier observations that the particle size for given polymers increases with polymer concentration. ${ }^{21,24,33,36}$ (iii) Charged groups, finally, could contribute to the reduction in surface tension, but should also be very effective in preventing aggregation. Furthermore, they could slow down transport of (charged) polymers to the charged surface of growing particles and thus the particle growth. ${ }^{19,36}$ In any of these cases, a higher amount of charged groups would result in a reduction of the particle size. Besides charged groups, hydrophilic groups could also contribute to the stabilization of the NPs, possibly through the formation of loops protruding from the particle surface. The presence of increasing amounts of hydrophilic groups on the NP surface is supposed to be at the origin of the decreasing absolute $\zeta$-potential with increasing fraction of hydrophilic groups in the polymers.

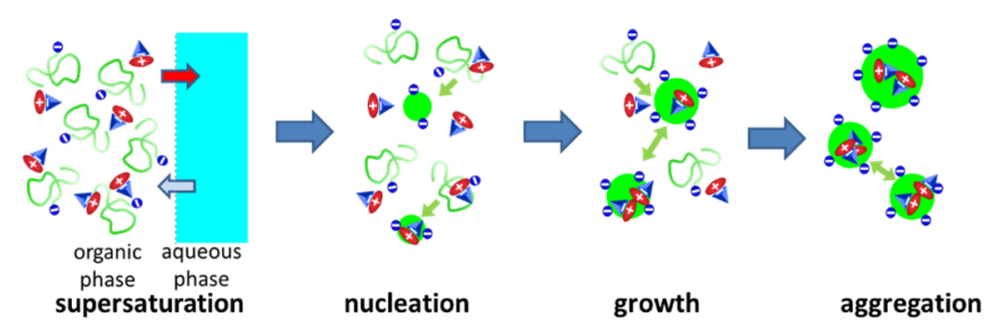

Figure 8. Schematic view of the process of nanoprecipitation of polymers and dye salt yielding dyeloaded polymer NPs: Upon adding the organic phase to the aqueous phase interdiffusion of the solvents occurs leading to supersaturation of the polymer and/or the dye salt. This triggers nucleation, followed by particle growth and possibly aggregation.

In the case of quantum yields, we observed a general trend of increasing quantum yield with increasing hydrophobicity of the used polymer (Figure 7B), but the increase was less clearly expressed than for the NP size. Indeed, on a global scale two large groups can be distinguished: (i) Polymers with a $\log \mathrm{P}<3$ gave particles with QYs between 10 and $35 \%$ that increased with increasing hydrophobicity. (ii) Polymers with $\log \mathrm{P}>3$ gave NPs with QYs around $60 \%$ that varied less clearly with hydrophobicity. Some crossover between these two populations exists, but these were typically the systems showing the largest variations from sample to sample.

Differences notably in the speed of precipitation of the dye salt and the polymer could explain such a behavior. ${ }^{16}$ Indeed, it has been shown that the dye salt alone can (nano)precipitate and even form NPs. ${ }^{41}$ For the most hydrophilic polymers, precipitation of the dye salt is expected to be faster than that of the polymers. This could then lead to clustering and 
aggregation of dyes and result in reduced QYs. A similar precipitation speed of both should on the other hand lead to a homogeneous distribution of the dyes in the matrix and higher QYs. The highest QYs would then correspond to the best matching of precipitation speeds. The 10 times higher amount of polymer is probably the reason that no clearly defined QY maximum, corresponding to the optimal speed matching, is observed, as the dye salt is simply integrated during polymer precipitation. Variations of the QYs in the second group might also come from distinct influences of the environment encountered by the dyes inside the particle and thus due to the specific chemical nature of the polymer.

\section{Conclusions}

Here we present a comprehensive study on the influence of the nature of the polymer on the formation of nanoparticles through nanoprecipitation. The composition of the polymers and in particular their global hydrophobicity and the presence of charged groups had a major influence on the sizes of the obtained particles, allowing to control them over a range from $\sim 10$ to $200 \mathrm{~nm}$. The specific chemical nature of the building blocks had, on the other hand, at most a minor influence on the particle size. The global hydrophobicity of the polymers also strongly influenced the photophysical properties, in particular the quantum yields, of fluorescent dyes encapsulated in the particles, which is linked to their modulated aggregation behavior. Both, size of particles and organization of load, are thus profoundly affected by the nature of the polymer. These effects are due to the influence of the polymer chemistry on nanoparticle formation in nanoprecipitation: Global hydrophobicity and the presence of hydrophilic and charged groups determine how the polymer behaves on addition of water, and thus should influence the kinetics of precipitation. Increasing hydrophobicity led to increasing size, probably due to variations of the ratio of nucleation and growth speeds, which controls the size distribution. At the same time, it led to increasing quantum yields of encapsulated dyes, probably through a better matching of the precipitation of polymer and dye salt. Polymer chemistry thus appears as a powerful tool for tailoring the properties of loaded polymer NPs, notably size and brightness of dye-loaded polymer NPs. The principles developed here should also be of highest interest for the design of aggregation induced emission nanoparticles and drug-delivery systems. 
Supporting Information. Details on the synthesized polymers (polymerization times, conversion, actual composition) and on the assembled NPs (size, $\zeta$-potential, spectra, QYs) are given in the

\section{Corresponding Author}

* Andreas Reisch

Laboratoire de Bioimagerie et Pathologies, CNRS UMR 7021

Université de Strasbourg - Faculté de Pharmacie

67401 Illkirch Cedex, France

E-mail: reisch@unista.fr

\section{Author Contributions}

The manuscript was written through contributions of all authors. All authors have given approval to the final version of the manuscript.

\section{Funding Sources}

Agence National de Recherche JC/JC grant "Supertrack" ANR-16-CE09-0007, European Research Council ERC Consolidator grant BrightSens 648528.

\section{Acknowledgements}

This work was supported by the Agence National de Recherche JC/JC grant "Supertrack" ANR16-CE09-0007 and by the European Research Council ERC Consolidator grant BrightSens 648528. The authors thank M. Legros and C. Foussat for size exclusion chromatography and C. Crucifix from the FRISBI platform (ANR-10-INBS-05) for help with electron microscopy.. 


\section{References}

(1) Horn, D.; Rieger, J. Organic Nanoparticles in the Aqueous Phase-Theory, Experiment, and Use. Angew. Chem. Int. Ed. 2001, 40 (23), 4330-4361. https://doi.org/10.1002/15213773(20011203)40:23<4330::AID-ANIE4330>3.0.CO;2-W.

(2) Elsabahy, M.; Heo, G. S.; Lim, S.-M.; Sun, G.; Wooley, K. L. Polymeric Nanostructures for Imaging and Therapy. Chem. Rev. 2015, 115 (19), 10967-11011. https://doi.org/10.1021/acs.chemrev.5b00135.

(3) Kamaly, N.; Yameen, B.; Wu, J.; Farokhzad, O. C. Degradable Controlled-Release Polymers and Polymeric Nanoparticles: Mechanisms of Controlling Drug Release. Chem. Rev. 2016, 116 (4), 2602-2663. https://doi.org/10.1021/acs.chemrev.5b00346.

(4) Ramasamy, T.; Ruttala, H. B.; Gupta, B.; Poudel, B. K.; Choi, H.-G.; Yong, C. S.; Kim, J. O. Smart Chemistry-Based Nanosized Drug Delivery Systems for Systemic Applications: A Comprehensive Review. J. Controlled Release 2017, 258, 226-253. https://doi.org/10.1016/j.jconrel.2017.04.043.

(5) Li, N.; Zhao, L.; Qi, L.; Li, Z.; Luan, Y. Polymer Assembly: Promising Carriers as CoDelivery Systems for Cancer Therapy. Prog. Polym. Sci. 2016, 58, 1-26. https://doi.org/10.1016/j.progpolymsci.2015.10.009.

(6) Oh, J. K.; Park, J. M. Iron Oxide-Based Superparamagnetic Polymeric Nanomaterials: Design, Preparation, and Biomedical Application. Prog. Polym. Sci. 2011, 36 (1), 168-189. https://doi.org/10.1016/j.progpolymsci.2010.08.005.

(7) Wolfbeis, O. S. An Overview of Nanoparticles Commonly Used in Fluorescent Bioimaging. Chem. Soc. Rev. 2015, 44, 4743-4768. https://doi.org/10.1039/C4CS00392F.

(8) Chen, M.; Yin, M. Design and Development of Fluorescent Nanostructures for Bioimaging. Prog. Polym. Sci. 2014, $39 \quad$ (2), 365-395. https://doi.org/10.1016/j.progpolymsci.2013.11.001.

(9) Rao, J. P.; Geckeler, K. E. Polymer Nanoparticles: Preparation Techniques and SizeControl Parameters. Prog. Polym. Sci. 2011, 36 (7), 887-913. https://doi.org/10.1016/j.progpolymsci.2011.01.001.

(10) Lepeltier, E.; Bourgaux, C.; Couvreur, P. Nanoprecipitation and the "Ouzo Effect": Application to Drug Delivery Devices. Adv. Drug Deliv. Rev. 2014, 71, 86-97. https://doi.org/10.1016/j.addr.2013.12.009.

(11) Saad, W. S.; Prud'homme, R. K. Principles of Nanoparticle Formation by Flash Nanoprecipitation. Nano Today 2016, 11 (2), 212-227. https://doi.org/10.1016/j.nantod.2016.04.006.

(12) Martínez Rivas, C. J.; Tarhini, M.; Badri, W.; Miladi, K.; Greige-Gerges, H.; Nazari, Q. A.; Galindo Rodríguez, S. A.; Román, R. Á.; Fessi, H.; Elaissari, A. Nanoprecipitation Process: From 
Encapsulation to Drug Delivery. Int. J. Pharm. 2017, 532 (1), 66-81. https://doi.org/10.1016/j.ijpharm.2017.08.064.

(13) Li, K.; Liu, B. Polymer-Encapsulated Organic Nanoparticles for Fluorescence and Photoacoustic Imaging. Chem. Soc. Rev. 2014, 43 (18), 6570-6597. https://doi.org/10.1039/C4CS00014E.

(14) Reisch, A.; Klymchenko, A. S. Fluorescent Polymer Nanoparticles Based on Dyes: Seeking Brighter Tools for Bioimaging. Small 2016, 12 (15), 1968-1992. https://doi.org/10.1002/smll.201503396.

(15) Gu, X.; Kwok, R. T. K.; Lam, J. W. Y.; Tang, B. Z. AIEgens for Biological Process Monitoring and Disease Theranostics. Biomaterials 2017, 146, 115-135. https://doi.org/10.1016/j.biomaterials.2017.09.004.

(16) Reisch, A.; Trofymchuk, K.; Runser, A.; Fleith, G.; Rawiso, M.; Klymchenko, A. S. Tailoring Fluorescence Brightness and Switching of Nanoparticles through Dye Organization in the Polymer Matrix. ACS Appl. Mater. Interfaces 2017, 9 (49), 43030-43042. https://doi.org/10.1021/acsami.7b12292.

(17) Reisch, A.; Didier, P.; Richert, L.; Oncul, S.; Arntz, Y.; Mély, Y.; Klymchenko, A. S. Collective Fluorescence Switching of Counterion-Assembled Dyes in Polymer Nanoparticles. Nat. Commun. 2014, 5, 4089. https://doi.org/10.1038/ncomms5089.

(18) Andreiuk, B.; Reisch, A.; Lindecker, M.; Follain, G.; Peyriéras, N.; Goetz, J. G.; Klymchenko, A. S. Fluorescent Polymer Nanoparticles for Cell Barcoding In Vitro and In Vivo. Small 2017, 13 (38), 1701582. https://doi.org/10.1002/smll.201701582.

(19) Reisch, A.; Heimburger, D.; Ernst, P.; Runser, A.; Didier, P.; Dujardin, D.; Klymchenko, A. S. Protein-Sized Dye-Loaded Polymer Nanoparticles for Free Particle Diffusion in Cytosol. Adv. Funct. Mater. 2018, 28 (48), 1805157. https://doi.org/10.1002/adfm.201805157.

(20) Trofymchuk, K.; Reisch, A.; Didier, P.; Fras, F.; Gilliot, P.; Mely, Y.; Klymchenko, A. S. Giant Light-Harvesting Nanoantenna for Single-Molecule Detection in Ambient Light. Nat. Photonics 2017, 11 (10), 657. https://doi.org/10.1038/s41566-017-0001-7.

(21) Lince, F.; Marchisio, D. L.; Barresi, A. A. Strategies to Control the Particle Size Distribution of Poly- $\varepsilon$-Caprolactone Nanoparticles for Pharmaceutical Applications. J. Colloid Interface Sci. 2008, 322 (2), 505-515. https://doi.org/10.1016/j.jcis.2008.03.033.

(22) D'Addio, S. M.; Prud'homme, R. K. Controlling Drug Nanoparticle Formation by Rapid Precipitation. Adv. Drug Deliv. Rev. 2011, 63 (6), 417-426. https://doi.org/10.1016/j.addr.2011.04.005.

(23) Beck-Broichsitter, M.; Nicolas, J.; Couvreur, P. Solvent Selection Causes Remarkable Shifts of the "Ouzo Region" for Poly(Lactide-Co-Glycolide) Nanoparticles Prepared by Nanoprecipitation. Nanoscale 2015, 7 (20), 9215-9221. https://doi.org/10.1039/C5NR01695A. 
Aubry, J.; Ganachaud, F.; Cohen Addad, J.-P.; Cabane, B. Nanoprecipitation of Polymethylmethacrylate by Solvent Shifting:1. Boundaries. Langmuir 2009, 25 (4), 1970-1979. https://doi.org/10.1021/la803000e.

(25) Johnson, B. K.; Prud'homme, R. K. Chemical Processing and Micromixing in Confined Impinging Jets. AIChE J. 2003, 49 (9), 2264-2282. https://doi.org/10.1002/aic.690490905.

(26) Jara, M. O.; Catalan-Figueroa, J.; Landin, M.; Morales, J. O. Finding Key Nanoprecipitation Variables for Achieving Uniform Polymeric Nanoparticles Using Neurofuzzy Logic Technology. Drug Deliv. Transl. Res. 2018, 8 (6), 1797-1806. https://doi.org/10.1007/s13346-0170446-8.

(27) Khan, S. A.; Schneider, M. Improvement of Nanoprecipitation Technique for Preparation of Gelatin Nanoparticles and Potential Macromolecular Drug Loading. Macromol. Biosci. 2013, 13 (4), 455-463. https://doi.org/10.1002/mabi.201200382.

(28) Contado, C.; Vighi, E.; Dalpiaz, A.; Leo, E. Influence of Secondary Preparative Parameters and Aging Effects on PLGA Particle Size Distribution: A Sedimentation Field Flow Fractionation Investigation. Anal. Bioanal. Chem. 2013, 405 (2-3), 703-711. https://doi.org/10.1007/s00216-012-6113-5.

(29) Geng, J.; Li, K.; Qin, W.; Ma, L.; Gurzadyan, G. G.; Tang, B. Z.; Liu, B. Eccentric Loading of Fluorogen with Aggregation-Induced Emission in PLGA Matrix Increases Nanoparticle Fluorescence Quantum Yield for Targeted Cellular Imaging. Small Weinh. Bergstr. Ger. 2013, 9 (11), 2012-2019. https://doi.org/10.1002/smll.201202505.

(30) Galindo-Rodriguez, S.; Allemann, E.; Fessi, H.; Doelker, E. Physicochemical Parameters Associated with Nanoparticle Formation in the Salting-out, Emulsification-Diffusion, and Nanoprecipitation Methods. Pharm. Res. 2004, $21 \quad$ (8), 1428-1439. https://doi.org/10.1023/B:PHAM.0000036917.75634.be.

(31) de Oliveira, A. M.; Jäger, E.; Jäger, A.; Stepánek, P.; Giacomelli, F. C. Physicochemical Aspects behind the Size of Biodegradable Polymeric Nanoparticles: A Step Forward. Colloids Surf. Physicochem. Eng. Asp. 2013, 436, 1092-1102. https://doi.org/10.1016/j.colsurfa.2013.08.056.

(32) Bilati, U.; Allémann, E.; Doelker, E. Development of a Nanoprecipitation Method Intended for the Entrapment of Hydrophilic Drugs into Nanoparticles. Eur. J. Pharm. Sci. 2005, 24 (1), 67-75. https://doi.org/10.1016/j.ejps.2004.09.011.

(33) Beck-Broichsitter, M.; Rytting, E.; Lebhardt, T.; Wang, X.; Kissel, T. Preparation of Nanoparticles by Solvent Displacement for Drug Delivery: A Shift in the "Ouzo Region” upon Drug Loading. Eur. J. Pharm. Sci. 2010, 41 (2), 244-253. https://doi.org/10.1016/j.ejps.2010.06.007.

(34) Legrand, P.; Lesieur, S.; Bochot, A.; Gref, R.; Raatjes, W.; Barratt, G.; Vauthier, C. Influence of Polymer Behaviour in Organic Solution on the Production of Polylactide Nanoparticles by 
$\begin{array}{lllllll}\text { Nanoprecipitation. } & \text { Int. } & \text { J. } & \text { Pharm. } & \text { 2007, } & 344 & \text { (1-2), }\end{array}$ https://doi.org/10.1016/j.ijpbarm.2007.05.054.

(35) Stepanyan, R.; Lebouille, J. G. J. L.; Slot, J. J. M.; Tuinier, R.; Stuart, M. A. C. Controlled Nanoparticle Formation by Diffusion Limited Coalescence. Phys. Rev. Lett. 2012, 109 (13), 138301. https://doi.org/10.1103/PhysRevLett.109.138301.

(36) Reisch, A.; Runser, A.; Arntz, Y.; Mély, Y.; Klymchenko, A. S. Charge-Controlled Nanoprecipitation as a Modular Approach to Ultrasmall Polymer Nanocarriers: Making Bright and Stable Nanoparticles. ACS Nano 2015, 9 (5), 5104-5116. https://doi.org/10.1021/acsnano.5b00214.

(37) Würth, C.; Geißler, D.; Behnke, T.; Kaiser, M.; Resch-Genger, U. Critical Review of the Determination of Photoluminescence Quantum Yields of Luminescent Reporters. Anal. Bioanal. Chem. 2014, 407 (1), 59-78. https://doi.org/10.1007/s00216-014-8130-z.

(38) Würth, C.; Grabolle, M.; Pauli, J.; Spieles, M.; Resch-Genger, U. Comparison of Methods and Achievable Uncertainties for the Relative and Absolute Measurement of Photoluminescence Quantum Yields. Anal. Chem. 2011, 83 (9), 3431-3439. https://doi.org/10.1021/ac2000303.

(39) Andreiuk, B.; Reisch, A.; Bernhardt, E.; Klymchenko, A. S. Fighting AggregationCaused Quenching and Leakage of Dyes in Fluorescent Polymer Nanoparticles: Universal Role of Counterion. Chem. - Asian J. 2019. https://doi.org/10.1002/asia.201801592R1.

(40) Mahajan, A. J.; Kirwan, D. J. Nucleation and Growth Kinetics of Biochemicals Measured at High Supersaturations. J. Cryst. Growth 1994, 144 (3), 281-290. https://doi.org/10.1016/0022-0248(94)90468-5.

(41) Shulov, I.; Oncul, S.; Reisch, A.; Arntz, Y.; Collot, M.; Mely, Y.; Klymchenko, A. S. Fluorinated Counterion-Enhanced Emission of Rhodamine Aggregates: Ultrabright Nanoparticles for Bioimaging and Light-Harvesting. Nanoscale 2015, $7 \quad$ (43), 18198-18210. https://doi.org/10.1039/C5NR04955E. 
Table of Contents Graphic

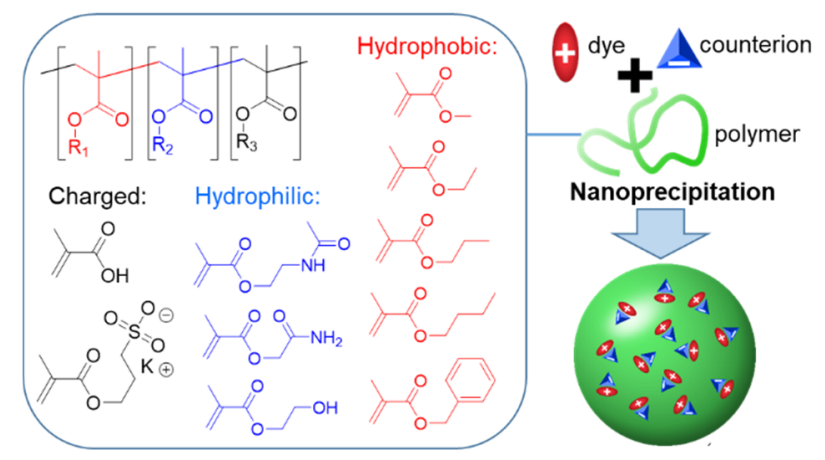

